\title{
Co-expression of tissue kallikrein 1 and tissue inhibitor of matrix metalloproteinase 1 improves myocardial ischemia-reperfusion injury by promoting angiogenesis and inhibiting oxidative stress
}

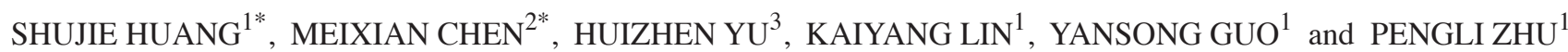 \\ ${ }^{1}$ Department of Cardiology, Fujian Provincial Hospital, Fuzhou, Fujian 350001; ${ }^{2}$ Department of Cardiology, \\ The 900th Hospital of Joint Logistics Support Force of People's Liberation Army, Fuzhou, Fujian 350025; \\ ${ }^{3}$ Department of Cardiology, Fujian Provincial Hospital Jinshan Branch, Fuzhou, Fujian 350028, P.R. China
}

Received July 15, 2020; Accepted November 23, 2020

DOI: $10.3892 / \mathrm{mmr} .2020 .11805$

\begin{abstract}
Myocardial ischemia/reperfusion (I/R) injury is a serious complication of reperfusion therapy for myocardial infarction. At present, there is not an effective treatment strategy available for myocardial I/R. The present study aimed to investigate the effects of human tissue kallikrein 1 (hTK1) and human tissue inhibitors of matrix metalloproteinase 1 (hTIMP1) gene co-expression on myocardial $\mathrm{I} / \mathrm{R}$ injury. A rat model of myocardial $\mathrm{I} / \mathrm{R}$ injury and a cell model with hypoxia/reoxygenation $(\mathrm{H} / \mathrm{R})$ treatment in cardiac microvascular endothelial cells (CMVECs) were established, and treated with adenovirus (Ad)-hTK1/hTIMP1. Following which, histological and triphenyl-tetrazolium-chloride staining assays were performed. Cardiac function was tested by echocardiographic measurement. The serum levels of oxidative stress biomarkers in rats and the intracellular reactive
\end{abstract}

Correspondence to: Professor Pengli Zhu or Professor Yansong Guo, Department of Cardiology, Fujian Provincial Hospital, 134 East Street, Gulou, Fuzhou, Fujian 350001, P.R. China

E-mail: huangshj125@sina.com

E-mail: ysguo1234@126.com

"Contributed equally

Abbreviations: AMI, acute myocardial infarction; CAT, catalase; CMVECs, cardiac microvascular endothelial cells; ECM, extracellular matrix; GSH, glutathione; GST, glutathioneS-transferase; H\&E, haematoxylin and eosin; H/R, hypoxia/ reoxygenation; I/R, ischemia/reperfusion; LVEF, left ventricular ejection fraction; LVFS, left ventricular fractional shortening; MDA, malondialdehyde; MMPs, matrix metallopeptidases; ROS, reactive oxygen species; SOD, superoxide dismutase; TIMPs, tissue inhibitors of matrix metalloproteinases; TK1, tissue kallikrein 1; TTC, triphenyl-tetrazolium-chloride; vWF, von Willebrand Factor

Key words: tissue kallikrein 1, tissue inhibitors of matrix metalloproteinase 1, myocardial infarction, ischemia-reperfusion injury, angiogenesis, oxidative stress oxygen species (ROS) levels in CMVECs were measured. Additionally, experiments, including immunostaining, reverse transcription-quantitative PCR, western blotting, and MTT, wound healing, Transwell and tube formation assays were also performed. The results of the present study demonstrated that Ad-hTK1/hTIMP1 alleviated myocardial injury and improved cardiac function in myocardial $\mathrm{I} / \mathrm{R}$ model rats. Ad-hTK1/hTIMP1 also significantly enhanced microvessel formation, decreased matrix metalloproteinase (MMP)2 and MMP9 expression, and reduced oxidative stress in myocardial I/R model rats. Furthermore, Ad-hTK1/hTIMP1 significantly enhanced proliferation, migration and tube formation in H/R-treated CMVECs. Additionally, Ad-hTK1/hTIMP1 significantly decreased intracellular ROS production and $\gamma$-H2A.X variant histone expression levels in H/R-treated CMVECs. In conclusion, the results of the present study demonstrated that co-expression of hTK1 and hTIMP1 genes displayed significant protective effects on myocardial I/R injury by promoting angiogenesis and suppressing oxidative stress; therefore, co-expression of hTK1 and hTIMP1 may serve as a potential therapeutic strategy for myocardial I/R injury.

\section{Introduction}

Acute myocardial infarction (AMI) is a subset of acute coronary syndrome that displays high morbidity and mortality (1). In the United States, the estimated annual incidence of AMI is 605,000 new cases and 200,000 recurrent cases, and the mortality is $\sim 14 \%$ (2). At present, the treatment strategies for AMI primarily include thrombolytic therapy and primary percutaneous coronary interventions (3). Although blood perfusion in the ischemic region is often rapidly recovered following immediate therapy, recanalization can aggravate myocardial injury, which is known as ischemia/reperfusion (I/R) injury (4). It has been suggested that myocardial I/R injury may decrease the benefits of myocardial reperfusion and worsen clinical outcomes (5). However, to the best of our knowledge, no effective treatment strategy for myocardial $\mathrm{I} / \mathrm{R}$ injury has been developed to date (6). Therefore, the discovery of novel effective therapeutic strategies for myocardial I/R injury is important. 
Tissue kallikrein 1 (TK1), a glycoprotein that belongs to the serine proteinase superfamily, can cleave lowmolecular-weight kininogen into a number of bioactive kinin peptides, including kininogens and kallikreins (7). Vasoactive kinins serve a beneficial role in a number of cardiovascular, cerebrovascular and renal diseases (8). A previous study revealed that hTK1 gene-modified mesenchymal stem cells display protective effects against cardiac injury following MI via suppressing apoptosis and inflammation, and promoting neovascularization (9). However, the functional role of TK1 in myocardial I/R injury requires further investigation.

Matrix metallopeptidases (MMPs) are a family of proteinases that possess the ability to degrade and remodel the extracellular matrix (ECM) (10). Adverse ECM remodeling is one of the key pathological processes in myocardial I/R injury (11). MMP activities are inhibited by tissue inhibitors of matrix metalloproteinases (TIMPs), of which there are four subtypes, TIMP1-4 (12). TIMP1 may serve as a plasma biomarker for predicting prognosis following MI (13). However, to the best of our knowledge, the potential role of TIMP1 in myocardial I/R injury has not been previously reported. Our previous study successfully constructed an adenovirus (Ad) vector containing hTK1 and hTIMP1 genes (14). In the present study, the beneficial effects of the co-expression of hTK1 and hTIMP1 genes on myocardial I/R injury in vivo and in vitro were assessed using the aforementioned Ad vector.

\section{Materials and methods}

Animals and treatments. A total of 30 male Sprague-Dawley rats (weight, 200-220 g; age, 4-6 weeks) were purchased from Beijing Vital River Laboratory Animal Technology Co., Ltd.. Rats were randomly divided into the following three groups ( $n=10$ per group): i) Sham; ii) I/R; and iii) $\mathrm{I} / \mathrm{R}+$ Ad-hTK1/hTIMP1. All rats were housed in an environment of the constant temperature $\left(22 \pm 2^{\circ} \mathrm{C}\right)$ and humidity $(55 \pm 5 \%)$ with 12 -h light/dark cycles, and ad libitum access to food and water. At 1 week prior to myocardial I/R injury, rats in the I/R + Ad-hTK1/hTIMP1 group were intravenously injected with recombinant Ad vector containing hTK1 and hTIMP1 genes (Ad-hTK1/hTIMP1; $5 \times 10^{12} \mathrm{gc} / \mathrm{kg}$ ). At the same time point, rats in the I/R and sham groups were intravenously injected with the control vector (Ad-EGFP; $5 \times 10^{12} \mathrm{gc} / \mathrm{kg}$ ). The Ad-hTK1/hTIMP1 and Ad-EGFP were constructed and maintained in our laboratory (Fujian Provincial Hospital Key Laboratory of Geriatrics, Fuzhou, China). All animal experiments were conducted in accordance with the National Institutes of Health Guide for the Care and Use of Laboratory Animals (15), and approved by the Institutional Ethics Committee for Laboratory Animal Care of Fujian Provincial Hospital (approval no. K2019-01-038).

Cell culture and transfection. Rat cardiac microvascular endothelial cells (CMVECs; LifeLine Cell Technology, LLC) were cultured in Dulbecco's modified Eagle's medium(DMEM; Gibco; Thermo Fisher Scientific, Inc.) supplemented with 20\% fetal bovine serum (FBS; Gibco; Thermo Fisher Scientific, Inc.), $100 \mathrm{U} / \mathrm{ml}$ penicillin (Gibco; Thermo Fisher Scientific, Inc.) and $100 \mu \mathrm{g} / \mathrm{ml}$ streptomycin (Gibco; Thermo Fisher
Scientific, Inc.) at $37^{\circ} \mathrm{C}$ in an air sealed chamber. CMVECs were transfected with Ad-hTK1/hTIMP1 or the control vector (Ad-EGFP), according to a previous study (16).

Myocardial I/R injury protocol. The induction of myocardial $\mathrm{I} / \mathrm{R}$ injury in the present study was performed as previously described (17), with a few modifications. Briefly, rats were anesthetized by the intraperitoneal injection of pentobarbital sodium $(40 \mathrm{mg} / \mathrm{kg})$ and were mechanically ventilated. The heart was exposed via a thoracotomy and subsequent opening of the pericardium. The left anterior descending coronary artery was ligated with a slipknot using a 7-0 silk suture. Ischemia of the anterior wall of the left ventricle was confirmed by observation of ST-segment elevation on the electrocardiogram. The heart was immediately placed back into the thoracic cavity and the chest was closed using a 4-0 silk suture. Following ischemia for $30 \mathrm{~min}$, the slipknot was released to allow for myocardial reperfusion. Rats in the sham group underwent an identical procedure, but ligation of the left anterior descending coronary artery was not performed. At $24 \mathrm{~h}$ post-surgery, echocardiographic measurements were conducted and blood samples $(2 \mathrm{ml})$ were collected from the carotid artery of each rat. All rats were euthanized by the intraperitoneal injection of an overdose of pentobarbital sodium (120 mg/kg). Death was verified by cessation of spontaneous breathing and the heartbeat. Subsequently, cardiac tissues were collected for use in subsequent experiments.

Establishment of the cell model with hypoxia/reoxygenation $(H / R)$ treatment. The cell model with $\mathrm{H} / \mathrm{R}$ treatment was established as previously described (18). Briefly, CMVECs were cultured in serum- and glucose-free DMEM in a hypoxic chamber $\left(1 \% \mathrm{O}_{2}\right)$ at $37^{\circ} \mathrm{C}$ for $6 \mathrm{~h}$. Subsequently, CMVECs were maintained in DMEM containing 20\% FBS in normoxic conditions $\left(21 \% \mathrm{O}_{2}\right)$ at $37^{\circ} \mathrm{C}$ for $12 \mathrm{~h}$ for reoxygenation.

Histological staining. Rat cardiac tissues were fixed in $4 \%$ paraformaldehyde (Beyotime Institute of Biotechnology) at $4^{\circ} \mathrm{C}$ for $24 \mathrm{~h}$, embedded in paraffin and cut into $4 \mu \mathrm{m}$-thick sections. For haematoxylin and eosin (H\&E) staining, sections were stained with haematoxylin for 3-5 min and 1\% eosin for $5 \mathrm{~min}$ at room temperature. For Masson and Sirius red staining, sections were stained using the Masson's Trichrome Stain kit and the Sirius Red Stain kit (both Beijing Solarbio Science \& Technology Co., Ltd.) according to the manufacturer's protocol, respectively. Stained sections were viewed and imaged at x200 magnification using an inverted optical light microscope (Olympus Corporation).

Triphenyl-tetrazolium-chloride (TTC) staining. TTC staining was performed to measure the myocardial infarct size as previously described (19). Briefly, hearts were harvested and perfused with ice-cold PBS. Subsequently, hearts were cut into 2-mm-thick slices and incubated with $1 \%$ TTC (Sigma-Aldrich; Merck $\mathrm{KGaA}$ ) diluted in $0.9 \%$ sodium chloride for $30 \mathrm{~min}$ at $37^{\circ} \mathrm{C}$, followed by fixation with $4 \%$ paraformaldehyde at room temperature for $30 \mathrm{~min}$. Then, stained slices were placed under natural light and imaged using a Canon EOS 2000D digital camera. The infarct area was quantified using ImageJ v1.8.0 software (National Institutes of Health). 
Echocardiographic measurement. Rats were anaesthetized by the intraperitoneal injection of pentobarbital sodium (40 mg/kg) and fixed in the supine position. Subsequently, two cardiac function parameters, left ventricular fractional shortening (LVFS) and left ventricular ejection fraction (LVEF), were assessed using a transthoracic echocardiography system (VisualSonics, Inc.).

Immunofluorescence staining. CD31, a marker of endothelial cells, immunofluorescence staining was performed to detect microvessels. Rat cardiac tissues were rapidly frozen at $-20^{\circ} \mathrm{C}$ and cut into $6 \mu \mathrm{m}$-thick sections using a freezing microtome (Leica Microsystems GmbH). Sections were fixed in $4 \%$ paraformaldehyde at room temperature for $30 \mathrm{~min}$ and washed with PBS three times. Following blocking in Immunol Staining Blocking Buffer (Beyotime Institute of Biotechnology) at room temperature for $15 \mathrm{~min}$, sections were incubated with an anti-CD31 primary antibody (cat. no. ab222783; 1:100; Abcam) overnight at $37^{\circ} \mathrm{C}$. Then, sections were incubated with a FITC-labelled goat anti-rabbit IgG secondary antibody (cat. no. A0562; 1:200; Beyotime Institute of Biotechnology) at room temperature for $1 \mathrm{~h}$. Stained sections were observed and imaged at x200 magnification using a confocal laser-scanning microscope (Leica Microsystems $\mathrm{GmbH}$ ). The fluorescent density of microvessels was analysed using Image J v1.8.0 software.

Immunohistochemistry. Rat cardiac tissues were fixed in $4 \%$ paraformaldehyde (Beyotime Institute of Biotechnology) at $4^{\circ} \mathrm{C}$ for $24 \mathrm{~h}$, embedded in paraffin and cut into $4 \mu \mathrm{m}$-thick sections. Following blocking in Immunol Staining Blocking Buffer at room temperature for $15 \mathrm{~min}$, sections were incubated with an anti-von Willebrand Factor (vWF) primary antibody (cat. no. ab6994; 1:200; Abcam) overnight at $4^{\circ} \mathrm{C}$. Subsequently, sections were incubated with a biotinylated mouse anti-rabbit IgG secondary antibody (cat. no. bs-0295M; $1: 100$; BIOSS) at room temperature for $30 \mathrm{~min}$. Sections were stained with dyaminobenzidine (Beyotime Institute of Biotechnology) for $10 \mathrm{~min}$ and haematoxylin for 3-5 min at room temperature. Stained sections were visualized and imaged at x200 magnification using an inverted optical light microscope (Olympus Corporation).

Reverse transcription-quantitative PCR (RT-qPCR). Total RNA was extracted from rat cardiac tissues using TRIzol ${ }^{\circledR}$ reagent (Thermo Fisher Scientific, Inc.) according to the manufacturer's protocol. Total RNA was reverse transcribed into cDNA using the ReverTra Ace qPCR RT kit (Toyobo Life Science), according to the manufacturer's protocol. Subsequently, qPCR was performed using a SYBR Green PCR master mix (Takara Biotechnology Co., Ltd.) on a LightCycler 480 instrument (Roche Diagnostics). The following thermocycling conditions were used for qPCR: Initial denaturation at $95^{\circ} \mathrm{C}$ for $30 \mathrm{sec}$; and 40 cycles of amplification at $95^{\circ} \mathrm{C}$ for $5 \mathrm{sec}, 60^{\circ} \mathrm{C}$ for $20 \mathrm{sec}$ and $72^{\circ} \mathrm{C}$ for $10 \mathrm{sec}$. The following primers were used for qPCR: MMP2 forward, 5'-GGGAATGAGTACTGGGTCTATT-3' and reverse, 5'-CCA GTTAAAGGCAGCGTCTA-3'; MMP9 forward, 5'-GATCAG CCGGGAACGTATCT-3' and reverse, 5'-AACTACAACGCC AGAAGTAT-3'; GAPDH forward, 5'-AACTTGGCATCG
TGGAAGG-3' and reverse, 5'-GTGGATGCAGGGATGATG TTC-3'. mRNA expression levels were quantified using the $2^{-\Delta \Delta \mathrm{Cq}}$ method (20) and normalized to the internal reference gene GAPDH.

Western blotting. Total protein was isolated from rat cardiac tissues and CMVECs using RIPA buffer (Beyotime Institute of Biotechnology) containing $1 \%$ protease inhibitor. Protein concentrations were determined using the BCA kit (Beyotime Institute of Biotechnology). Proteins $(40 \mu \mathrm{g})$ were separated via 8 or $15 \%$ SDS-PAGE and subsequently transferred to PVDF membranes (MilliporeSigma). Following blocking in $5 \%$ skimmed milk at room temperature for $2 \mathrm{~h}$, the membranes were incubated overnight at $4^{\circ} \mathrm{C}$ with the following primary antibodies (all purchased from Abcam): Anti-TK1 (cat. no. ab28289; 1:1,000), anti-TIMP1 (cat. no. ab109125; 1:1,000), anti-MMP2 (cat. no. ab92536; 1:1,000), anti-MMP9 (cat. no. ab76003; 1:1,000), anti-GAPDH (cat. no. ab181602; 1:10,000), anti- $\gamma$-H2A.X (cat. no. ab81299; 1:5,000), anti-H2A.X (cat. no. ab11175; 1:1,000) and anti-TATA binding protein (TBP; cat. no. ab63766; 1:1,000). Subsequently, the membranes were incubated with a HRP-conjugated goat anti-rabbit IgG secondary antibody (cat. no. BA1054; 1:5,000; Wuhan Boster Biological Technology, Ltd.) at room temperature for $2 \mathrm{~h}$. Protein bands were visualized using an enhanced chemiluminescence kit (Thermo Fisher Scientific, Inc.) and a chemiluminescence imaging system (Bio-Rad Laboratories, Inc.). Protein expression levels were semi-quantified using ImageJ v1.8.0 software with GAPDH and TBP as the loading controls.

Assessment of oxidative stress. Following reperfusion for $24 \mathrm{~h}$, blood samples were collected from the carotid artery of anesthetized rats. To obtain serum samples, blood samples were maintained at room temperature for $2 \mathrm{~h}$ prior to centrifugation at $1,000 \mathrm{x}$ g for $20 \mathrm{~min}$ at $4^{\circ} \mathrm{C}$. Serum levels of superoxide dismutase (SOD), malondialdehyde (MDA), glutathione (GSH), catalase (CAT) and glutathione-S-transferase (GST) were measured using corresponding kits (SOD, cat. no. BC0175; MDA, cat. no. BC0025; GSH, cat. no. BC1175; CAT, cat. no. BC0205; GST, cat. no. BC0350; all purchased from Beijing Solarbio Science \& Technology Co., Ltd.) according to the manufacturer's protocols.

Following H/R treatment, intracellular ROS production in CMVECs was detected using CellROX Green reagent (Thermo Fisher Scientific, Inc.). Cells were incubated with $5 \mu \mathrm{M}$ CellROX Green reagent at $37^{\circ} \mathrm{C}$ for $30 \mathrm{~min}$ and washed three times with PBS. Subsequently, cells were incubated with DAPI (Beyotime Institute of Biotechnology) at room temperature for $10 \mathrm{~min}$. Stained cells were observed and imaged at x200 magnification using a confocal laser-scanning microscope (Leica Microsystems $\mathrm{GmbH}$ ). The ratio of green fluorescence/nuclei staining in each image was calculated.

Cell viability assay. CMVECs were seeded ( $1 \times 10^{3}$ cells/well) into 96-well plates and treated with H/R. Cells were washed with PBS and incubated with $0.5 \mathrm{mg} / \mathrm{ml} \mathrm{MTT} \mathrm{solution} \mathrm{at}$ $37^{\circ} \mathrm{C}$ for $4 \mathrm{~h}$. The incubation solution was discarded and the formazan crystals were dissolved in $150 \mu \mathrm{l}$ DMSO. The 
A
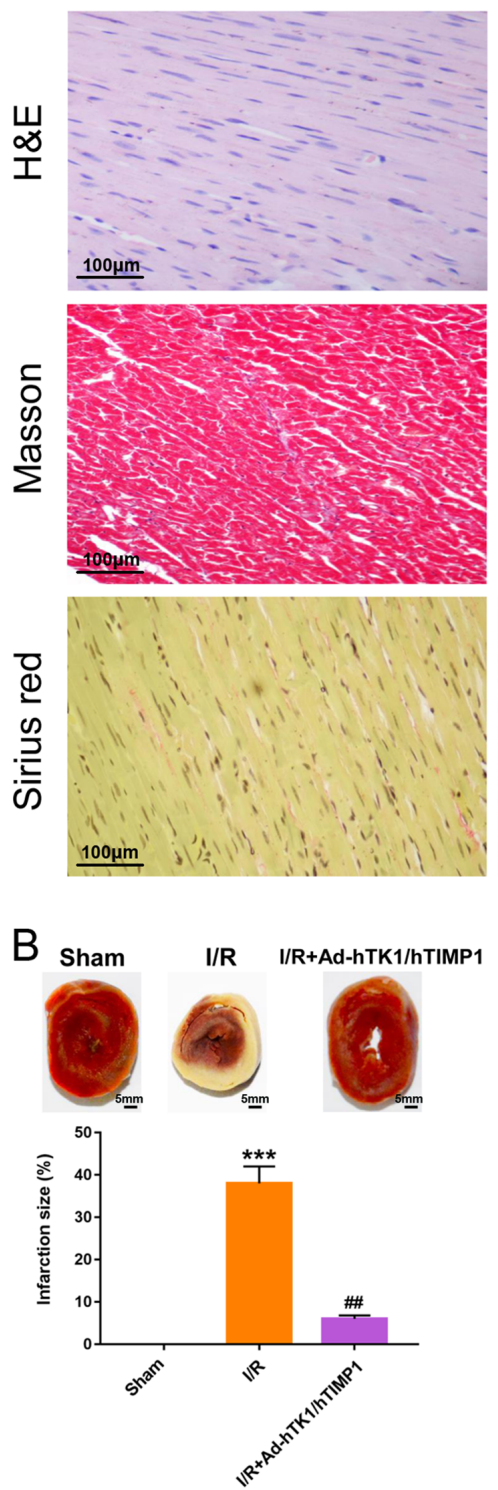

$\mathrm{I} / \mathrm{R}$
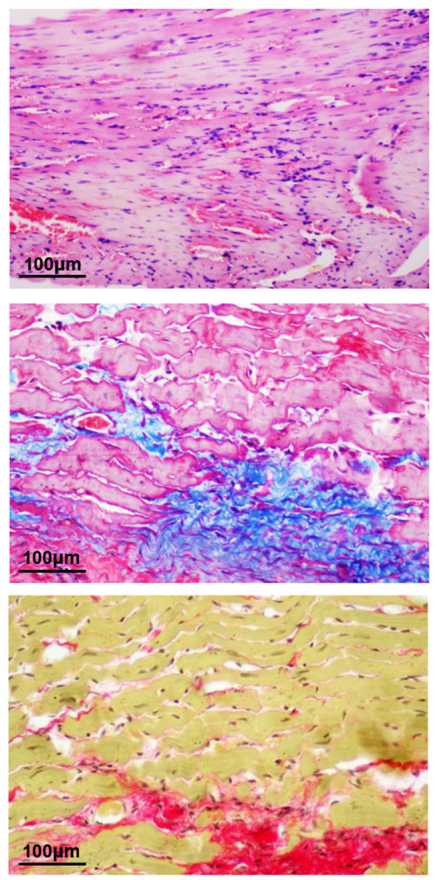

C

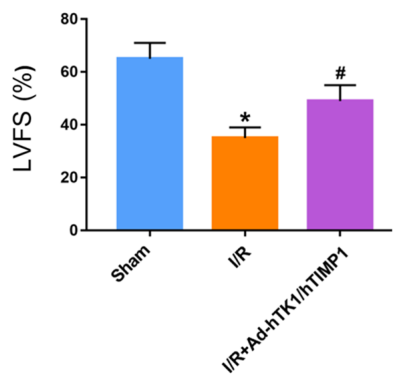

I/R+Ad-hTK1/hTIMP1
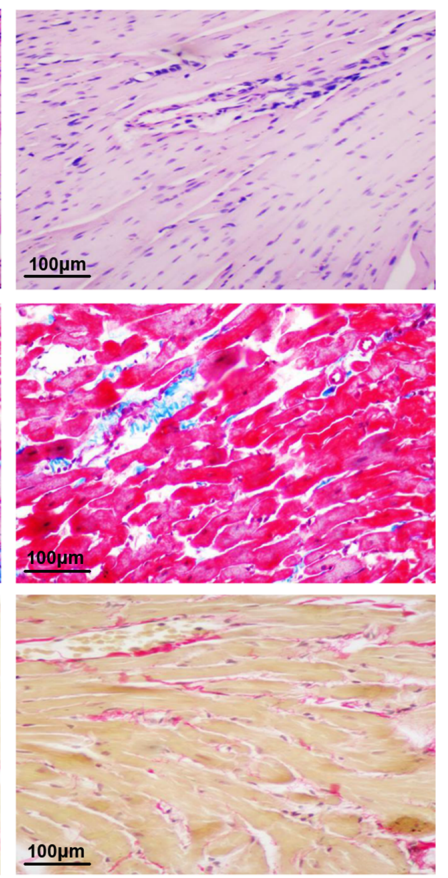

D

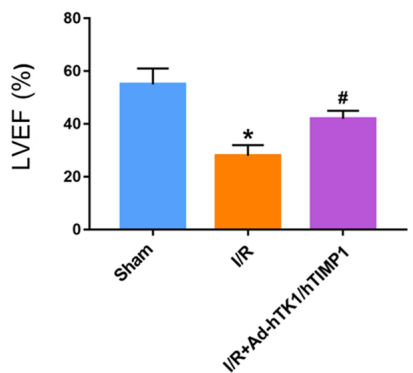

Figure 1. Ad-hTK1/hTIMP1 significantly ameliorates myocardial injury and improves cardiac function in myocardial I/R model rats. (A) H\&E, Masson and Sirius red staining were performed on cardiac tissue sections of rats in the sham, I/R and I/R + Ad-hTK1/hTIMP1 groups. Blue in Masson-stained sections and red in Sirius red-stained sections represent collagen deposition. Scale bar, $100 \mu \mathrm{m}$. (B) Myocardial infarction size was measured via triphenyl-tetrazolium-chloride staining. Scale bar, $5 \mathrm{~mm}$. Echocardiographic measurement of the two cardiac function parameters (C) LVFS and (D) LVEF. * $<0.05$ and ${ }^{* * * *} \mathrm{P}<0.001$ vs. Sham; ${ }^{\#} \mathrm{P}<0.05$ and ${ }^{\# \#} \mathrm{P}<0.01$ vs. I/R. Ad, adenovirus; hTK1, human tissue kallikrein 1; hTIMP1, human tissue inhibitors of matrix metalloproteinase 1; I/R, ischemia/reperfusion; LVFS, left ventricular fractional shortening; LVEF, left ventricular ejection fraction.

absorbance was measured at a wavelength of $570 \mathrm{~nm}$ using a microplate reader (Thermo Fisher Scientific, Inc.).

Wound healing assay. CMVECs were seeded ( $5 \times 10^{5}$ cells/well) into 6-well plates and cultured in FBS-free medium until cells reached $90 \%$ confluence. The cell monolayer was scraped using a sterile $200 \mu \mathrm{l}$ pipette tip and washed twice with sterile PBS. The remaining adherent cells were treated with $H / R$ and then cultured for $48 \mathrm{~h}$ in FBS-free medium. Images of the wound at x100 magnification were captured at 0 and $48 \mathrm{~h}$ using an inverted optical light microscope (Olympus Corporation).

Transwell assay. CMVECs $\left(5 \times 10^{4}\right)$ were seeded into the upper chambers of the Transwell inserts (pore size, $8 \mu \mathrm{m}$; EMD Millipore) with FBS-free medium. DMEM supplemented with $20 \%$ FBS was plated into the lower chambers. Following treatment with $\mathrm{H} / \mathrm{R}$ and cell culture for $48 \mathrm{~h}$, migratory cells were fixed in $4 \%$ paraformaldehyde at room temperature for $30 \mathrm{~min}$ and stained with $0.1 \%$ crystal violet (Beyotime Institute of Biotechnology) at room temperature for $15 \mathrm{~min}$. Following washing twice with PBS, migratory cells were observed and imaged at x200 magnification using an inverted optical light microscope (Olympus Corporation) and the number of cells was quantified for analysis.

Tube formation assay. For the tube formation assay, 96-well plates were coated with Matrigel (BD Biosciences) and incubated at $37^{\circ} \mathrm{C}$ for $30 \mathrm{~min}$. CMVECs were seeded (1x10 ${ }^{4}$ cells/well) into the 96 -well plates and treated with H/R. After culturing at $37^{\circ} \mathrm{C}$ for $24 \mathrm{~h}$, capillary-like tubes were 
A
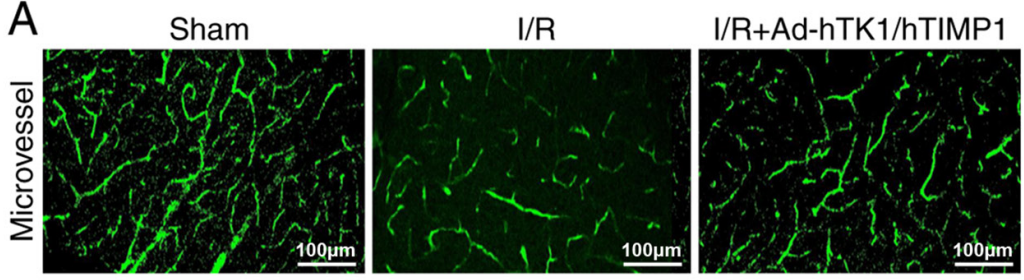

C
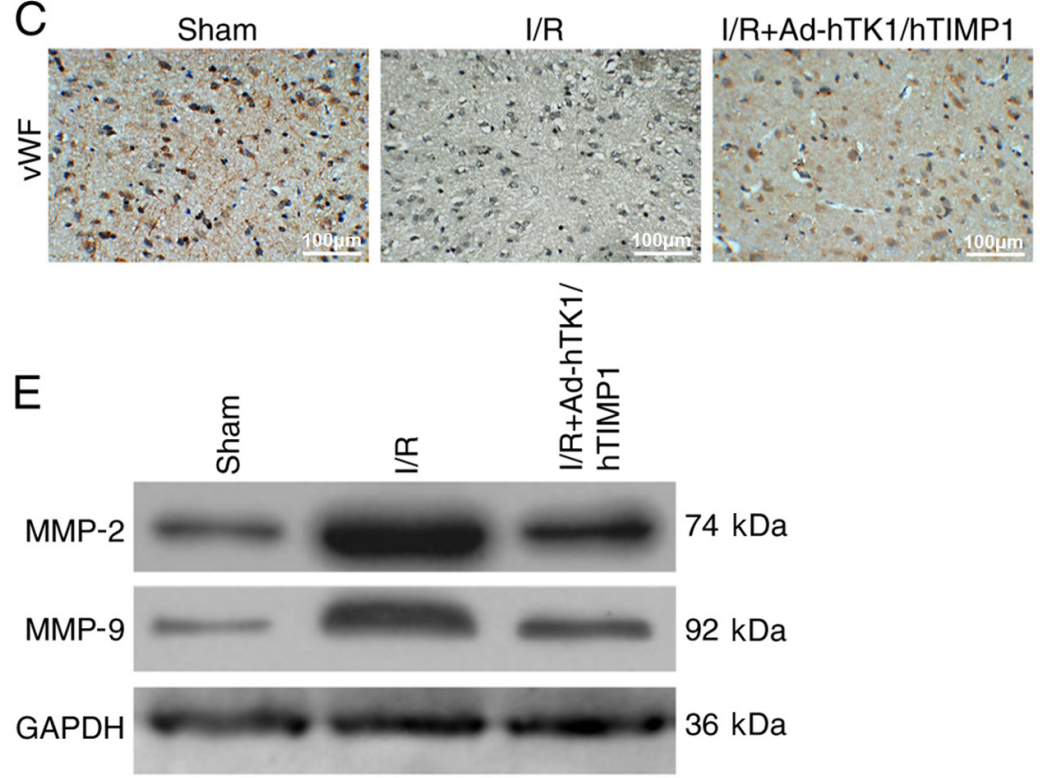
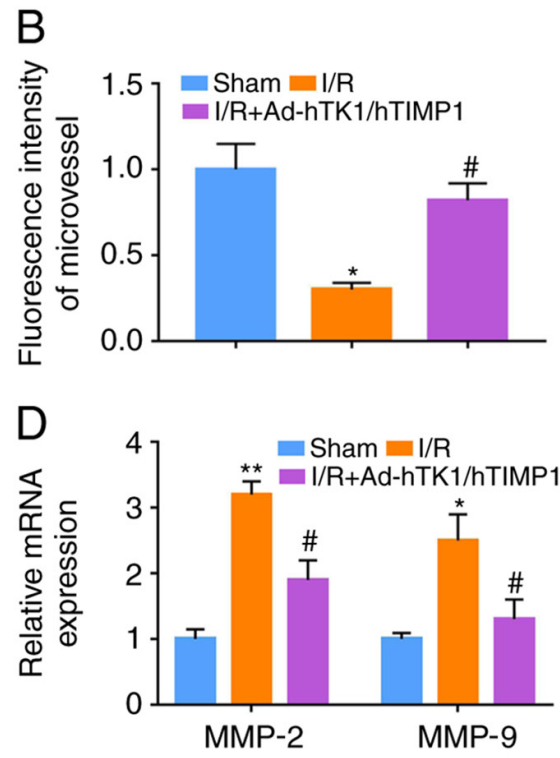

F

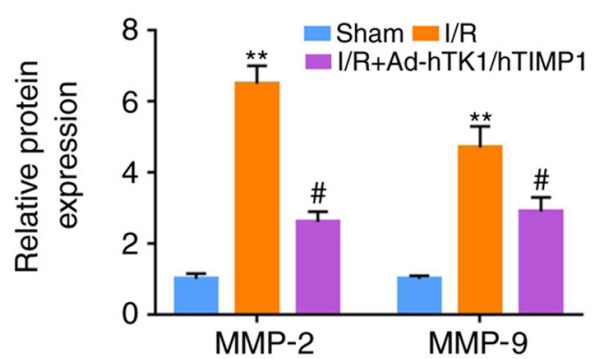

Figure 2. Ad-hTK1/hTIMP1 promotes microvessel formation, and reduces MMP2 and MMP9 expression levels in myocardial I/R model rats. Microvessels in the myocardium of rats in the sham, I/R and I/R + Ad-hTK1/hTIMP1 groups were (A) detected via immunofluorescence staining using an anti-CD31 primary antibody and (B) quantified. Scale bar, $100 \mu \mathrm{m}$. (C) Immunohistochemical staining of vWF-positive cells in the myocardium of rats in the sham, I/R and I/R + Ad-hTK1/hTIMP1 groups. Scale bar, $100 \mu \mathrm{m}$. (D) MMP2 and MMP9 mRNA expression levels in rat hearts. MMP2 and MMP9 protein expression levels in rat hearts were (E) determined via western blotting and (F) semi-quantified. ${ }^{*} \mathrm{P}<0.05$ and ${ }^{* *} \mathrm{P}<0.01$ vs. Sham; ${ }^{*} \mathrm{P}<0.05$ vs. I/R. Ad, adenovirus; hTK1, human tissue kallikrein 1; hTIMP1, human tissue inhibitors of matrix metalloproteinase 1; MMP, matrix metallopeptidase; I/R, ischemia/reperfusion; vWF, von Willebrand Factor.

observed and imaged at x200 magnification using an inverted optical light microscope (Olympus Corporation). Tube length was quantified using ImageJ v1.8.0 software.

Statistical analysis. Data are presented as the mean \pm SD. All experiments were conducted with at least three independent repeats. Comparisons among multiple groups were analysed using one-way ANOVA followed by Bonferroni's post hoc test. Statistical analyses were performed using SPSS software (version 22.0; IBM Corp.). $\mathrm{P}<0.05$ was considered to indicate a statistically significant difference.

\section{Results}

Ad-hTK1/hTIMP1 significantly ameliorates myocardial injury and improves cardiac function in myocardial $I / R$ model rats. Compared with the control vector, Ad-hTK1/hTIMP1 injection notably increased the protein expression levels of hTK1 and hTIMP1 in rat hearts (Fig. S1A), which demonstrated that hTK1 and hTIMP1 were expressed in rat hearts after gene delivery. Moreover, the H\&E staining results demonstrated that myocardial cells in sham rats were well-arranged and displayed intact muscle fibres, whereas myocardial cells were irregularly arranged and obvious necrosis was observed in the myocardium following myocardial I/R injury (Fig. 1A). Injection with Ad-hTK1/hTIMP1 partly ameliorated histopathological alterations in myocardial I/R model rats. Furthermore, the Masson and Sirius red staining results displayed obvious collagen deposition in the myocardium following myocardial $\mathrm{I} / \mathrm{R}$, which was notably reduced by Ad-hTK1/hTIMP1 (Fig. 1A). Furthermore, the results demonstrated that I/R treatment significantly increased myocardial infarct size compared with sham rats (Fig. 1B), whereas Ad-hTK1/hTIMP1 significantly decreased myocardial infarct size in myocardial I/R model rats. The results also demonstrated that LVFS and LVEF in myocardial $\mathrm{I} / \mathrm{R}$ model rats were significantly reduced compared with sham rats, but these alterations were significantly reversed by Ad-hTK1/hTIMP1 (Fig. 1C and D). The aforementioned results indicated that Ad-hTK1/hTIMP1 significantly alleviated myocardial injury and improved cardiac function in myocardial I/R model rats.

Ad-hTK1/hTIMP1 promotes microvessel formation, and reduces MMP2 and MMP9 expression levels in myocardial $I / R$ model rats. The microvessels in rat hearts were detected via CD31 immunofluorescence staining (Fig. 2A). 
A

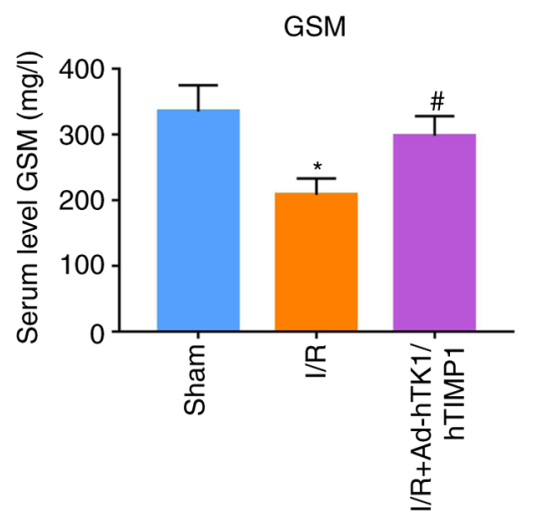

$\mathrm{B}$

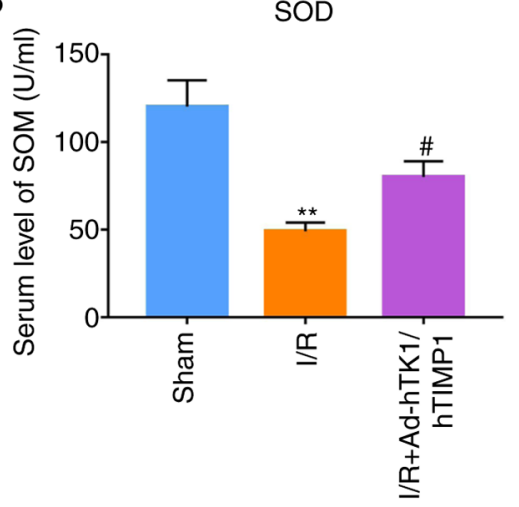

C

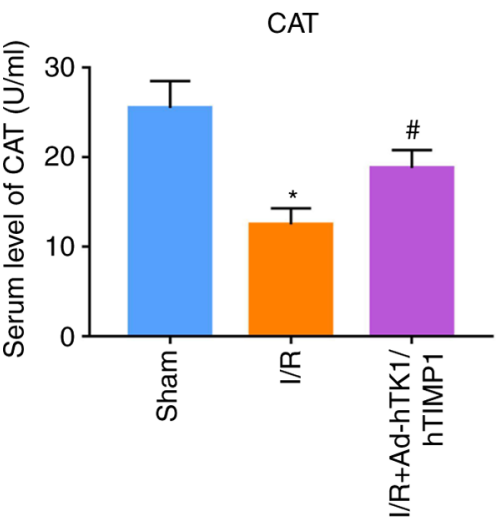

D

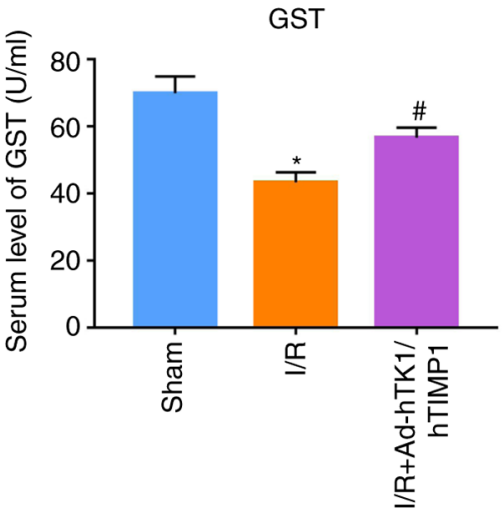

E

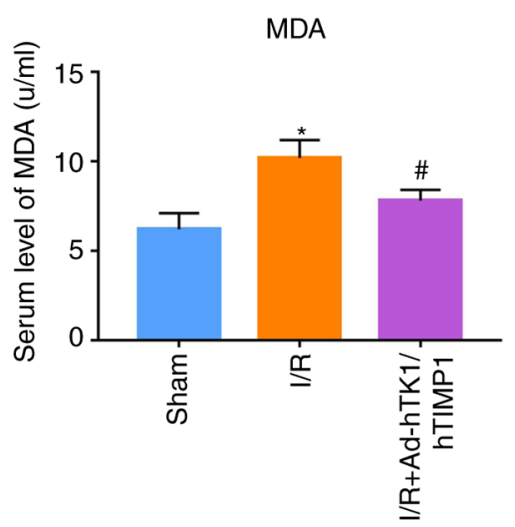

Figure 3. Ad-hTK1/hTIMP1 significantly reduces oxidative stress in myocardial I/R model rats. Measurement of serum levels of oxidative stress biomarkers, including (A) GSH, (B) SOD, (C) CAT, (D) GST and (E) MDA, in rats in the sham, I/R and I/R + Ad-hTK1/hTIMP1 groups. * $<0.05$ and ** $<0.01$ vs. Sham; ${ }^{\#} \mathrm{P}<0.05$ vs. I/R. Ad, adenovirus; hTK1, human tissue kallikrein 1; hTIMP1, human tissue inhibitors of matrix metalloproteinase 1; I/R, ischemia/reperfusion; GSH, glutathione; SOD, superoxide dismutase; CAT, catalase; GST, glutathione-S-transferase; MDA, malondialdehyde.

The fluorescence density of microvessels in the myocardium of myocardial I/R model rats was significantly lower compared with sham rats, and was significantly enhanced following Ad-hTK1/hTIMP1 treatment (Fig. 2B). Furthermore, Ad-hTK1/hTIMP1 markedly increased the number of vWF-positive cells in the myocardium of myocardial I/R model rats (Fig. 2C). The results also indicated that compared with the sham group, I/R treatment significantly increased MMP2 and MMP9 mRNA and protein expression levels, and Ad-hTK1/hTIMP1 significantly downregulated MMP2 and MMP9 expression levels in myocardial I/R model rats (Fig. 2D-F).

Ad-hTK1/hTIMP1 significantly reduces oxidative stress in myocardial $I / R$ model rats. Subsequently, serum levels of oxidative stress biomarkers were measured. The results indicated that GSH serum levels, and SOD, CAT and GST activities in myocardial $\mathrm{I} / \mathrm{R}$ model rats were significantly decreased compared with sham rats. However, Ad-hTK1/hTIMP1 significantly reversed I/R-induced effects on oxidative stress in myocardial I/R model rats (Fig. 3A-D). Compared with sham rats, the serum level of MDA in myocardial I/R model rats was significantly increased, which was significantly reduced by Ad-hTK1/hTIMP1 (Fig. 3E). The results indicated that Ad-hTK1/hTIMP1 significantly reduced oxidative stress in myocardial I/R model rats.
Ad-hTK1/hTIMP1 significantly enhances H/R-treated CMVEC proliferation, migration and tube formation. Following transfection with Ad-hTK1/hTIMP1, the protein expression levels of hTK1 and hTIMP1 in CMVECs were notably increased compared with the control vector (Fig. S1B), demonstrating the successful transfection of Ad-hTK1/hTIMP1 in CMVECs. Subsequently, the cell model with H/R treatment was established in CMVECs. Compared with the control group, CMVEC proliferation in the $\mathrm{H} / \mathrm{R}$ group was significantly suppressed, whereas Ad-hTK1/hTIMP1 significantly enhanced proliferation in H/R-treated CMVECs (Fig. 4A). Furthermore, $\mathrm{H} / \mathrm{R}$ treatment notably inhibited CMVEC migration at $48 \mathrm{~h}$ compared with the control group, and Ad-hTK1/hTIMP1 considerably attenuated the inhibitory effect of H/R treatment (Fig. 4B). Furthermore, the Transwell assay results demonstrated that compared with the control group, the number of migratory CMVECs was significantly decreased following treatment with $\mathrm{H} / \mathrm{R}$, which was significantly increased by Ad-hTK1/hTIMP1 (Fig. 4C and D). Additionally, compared with the control group, tube lengths in the H/R group were significantly reduced, which was significantly ameliorated by Ad-hTK1/hTIMP1 (Fig. 4E and F).

Ad-hTK1/hTIMP1 significantly decreases intracellular ROS production and $\gamma-H 2 A$.X expression in $H / R$-treated $C M V E C s$. The ratio of green fluorescence/nuclei staining of the $H / R$ group was significantly higher compared with the 

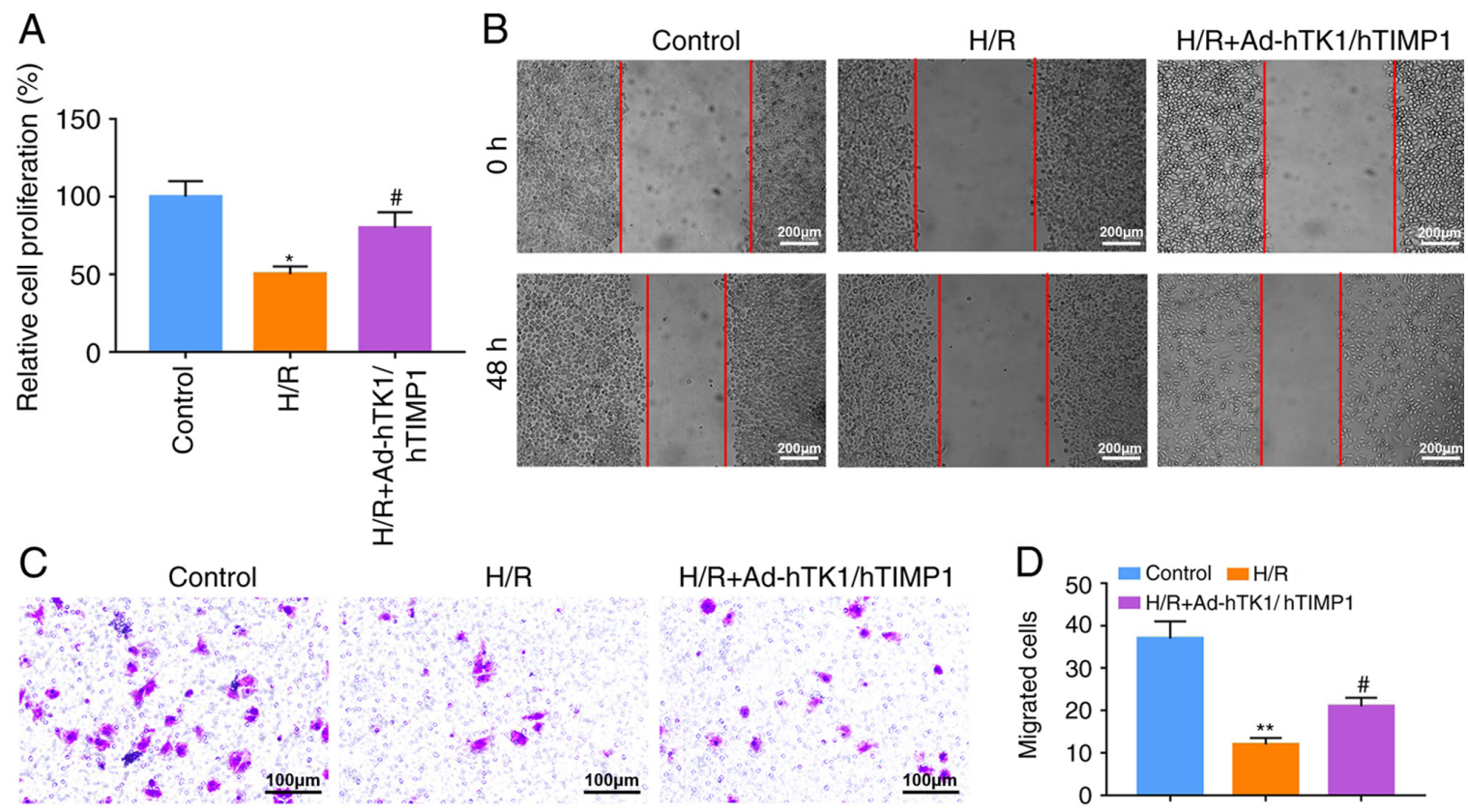

$\mathrm{E}$ Control
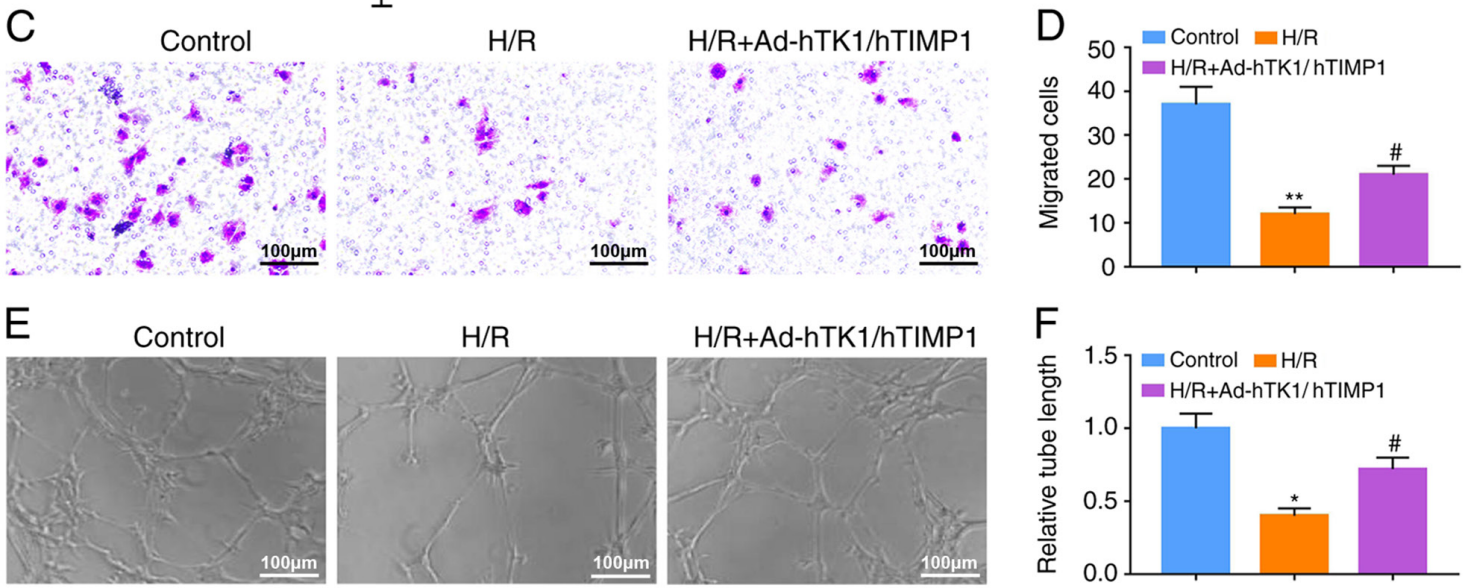

Figure 4. Ad-hTK1/hTIMP1 significantly enhances H/R-treated CMVEC proliferation, migration and tube formation. The cell model with H/R treatment was established in CMVECs. (A) CMVEC proliferation was detected by performing the MTT assay. (B) CMVEC migration was assessed by conducting wound healing assays. The area between the two red lines indicates the wound area. Scale bar, $200 \mu \mathrm{m}$. CMVEC migration was also (C) measured by performing a Transwell assay and (D) quantified. Scale bar, $100 \mu \mathrm{m}$. CMVEC tube formation was (E) assessed by conducting a tube formation assay and (F) quantified. Scale bar, $100 \mu \mathrm{m}$. ${ }^{*} \mathrm{P}<0.05$ and ${ }^{* *} \mathrm{P}<0.01$ vs. Control; ${ }^{*} \mathrm{P}<0.05$ vs. H/R. Ad, adenovirus; hTK1, human tissue kallikrein 1; hTIMP1, human tissue inhibitors of matrix metalloproteinase 1; H/R, hypoxia/reoxygenation; CMVEC, cardiac microvascular endothelial cells; I/R, ischemia/reperfusion.

A
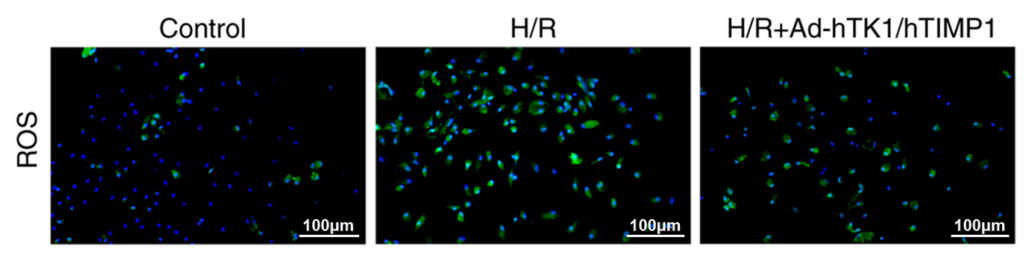

C

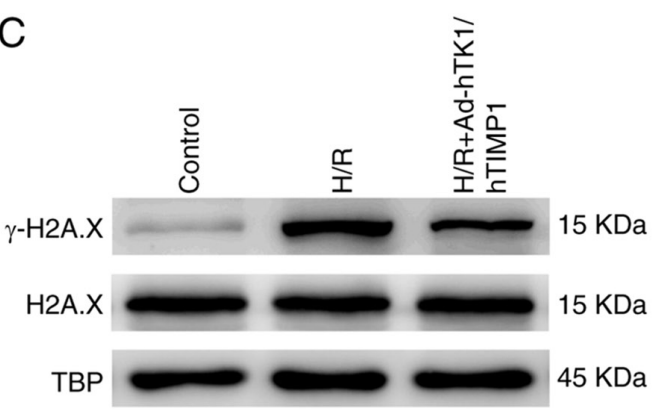

B

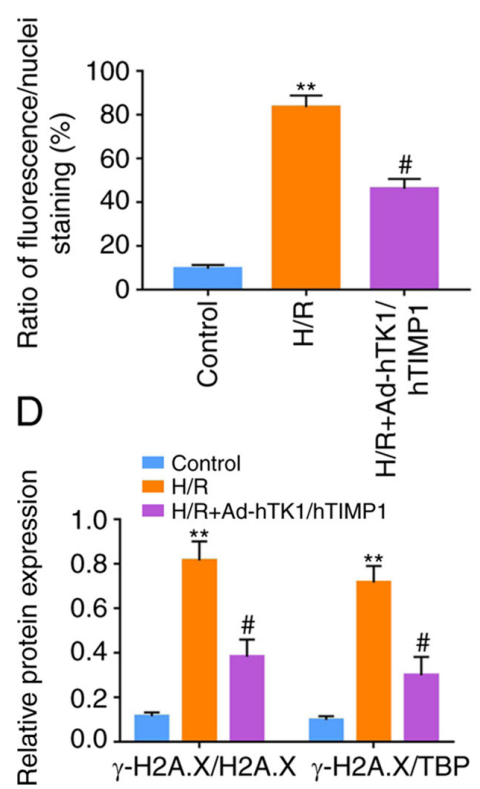

Figure 5. Ad-hTK1/hTIMP1 significantly decreases intracellular ROS production and $\gamma$-H2A.X expression levels in H/R-treated CMVECs. (A) Measurement of intracellular ROS (green fluorescence) in CMVECs. Nuclei were labelled using DAPI (blue fluorescence). Scale bar, $100 \mu \mathrm{m}$. (B) The ratio of green fluorescence/nuclei staining was quantified. $\gamma$-H2A.X and H2A.X protein expression levels in CMVECs were (C) determined via western blotting and (D) semi-quantified. ${ }^{* *} \mathrm{P}<0.01$ vs. Control; " $\mathrm{P}<0.05$ vs. H/R. Ad, adenovirus; hTK1, human tissue kallikrein 1; hTIMP1, human tissue inhibitors of matrix metalloproteinase 1; ROS, reactive oxygen species; H/R, hypoxia/reoxygenation; CMVEC, cardiac microvascular endothelial cells; H2A.X, H2A.X variant histone; TBP, TATA binding protein. 
control group, indicating that intracellular ROS production in CMVECs was significantly enhanced following $\mathrm{H} / \mathrm{R}$ treatment (Fig. 5A). However, Ad-hTK1/hTIMP1 significantly reduced intracellular ROS production in H/R-treated CMVECs (Fig. 5B). Furthermore, the relative protein expression levels of $\gamma$-H2A.X in H/R-treated CMVECs were significantly increased compared with the control group. By contrast, $\gamma$-H2A.X expression levels in H/R-treated CMVECs were significantly decreased by Ad-hTK1/hTIMP1 (Fig. 5C and D).

\section{Discussion}

Collagen deposition due to an imbalance of myocardial ECM deposition and degradation following myocardial I/R injury may lead to myocardial fibrosis, which may induce myocardial pathological remodeling and decrease cardiac function (21). MMPs and their inhibitors, TIMPs, have been reported to be vital regulators in ECM remodelling (22). A previous study reported that a substantial increase in MMP9 and MMP2 expression levels was positively correlated with the degree of left ventricular fibrosis in a mouse model of pressure overload (23). Additionally, a previous study demonstrated that MMP9 and MMP2 expression levels were significantly increased in myocardial I/R model mice compared with sham mice (24). In the present study, compared with sham rats, MMP9 and MMP2 mRNA and protein expression levels were significantly increased following myocardial I/R injury, but significantly reduced following Ad-hTK1/hTIMP1 injection. Meanwhile, a notable decrease in collagen deposition in the myocardium of myocardial I/R model rats following Ad-hTK1/hTIMP1 treatment was also observed in the present study, which may be attributed to exogenous TIMP1. In addition, a previous study reported that exogenous hTK1 gene delivery decreases infarct size and improves cardiac function following myocardial I/R injury by limiting post-infarct ventricular remodeling via inhibition of $\mathrm{NF}-\kappa \mathrm{B}$ activation (25). Furthermore, hTK1-modified mesenchymal stem cells have been demonstrated to exhibit enhanced beneficial effects on cardiac injury following MI, which are primarily manifested as promoting cardiomyocyte survival, inhibiting ventricular remodeling and improving cardiac function (9). The aforementioned results indicated the protective role of hTK1 gene against cardiac injury in ischemic heart diseases. Consistently, the present study indicated that Ad-hTK1/hTIMP1 significantly ameliorated myocardial injury, decreased infarct size and improved cardiac function following myocardial I/R injury. Therefore, the results suggested that Ad-hTK1/hTIMP1 displayed its protective effects of alleviating cardiac injury and preventing cardiac remodeling after myocardial I/R injury via the co-expression of hTK1 and hTIMP1 genes.

$\mathrm{I} / \mathrm{R}$ can cause vascular endothelial injury via a number of different mechanisms, including $\mathrm{pH}$ change induced cytotoxicity, oxidative stress and inhibition of endothelial nitric oxide synthase (26). According to the American College of Cardiology/American Heart Association guidelines, clinical treatments that minimize microvascular damage should be prioritized when attempting to protect the injured myocardium (27). Therefore, any treatment that can promote angiogenesis may be an effective therapeutic strategy for myocardial I/R injury. It has been previously reported that exogenous TK1 administration can significantly enhance vascular density in the peri-infarction region following myocardial infarction (28) or cerebral cortex infarction (29). TIMP1 has also been reported to facilitate blood vessel formation via inhibiting MMP9 in vitro (30). Consistently, the present study demonstrated that co-treatment of hTK1 and hTIMP1 genes significantly increased the microvessel density in the myocardium of myocardial $\mathrm{I} / \mathrm{R}$ model rats. It was also demonstrated that the number of vWF [a biomarker of angiogenesis (31)]-positive cells was significantly increased by Ad-hTK1/hTIMP1 in the myocardium of myocardial I/R model rats. Furthermore, in vitro experiments demonstrated that Ad-hTK1/hTIMP1 significantly ameliorated H/R-induced suppression of CMVEC proliferation and migration. Ad-hTK1/hTIMP1 also significantly increased tube formation in H/R-treated CMVECs. The results of the present study indicated the prominent effects of co-expression of hTK1 and hTIMP1 genes on promoting angiogenesis following I/R injury, which may serve a key role in improving myocardial I/R injury.

Oxidative stress is an important pathological process that contributes to myocardial I/R injury (32) and can damage all components of the cell, including DNA, proteins and lipids (33). Previous research has reported that any treatment that can reduce oxidative stress may attenuate myocardial I/R injury (34-36). It has been previously reported that hTK1 can improve erectile dysfunction of streptozotocin-induced diabetic model rats and attenuate salt-induced renal fibrosis via inhibition of oxidative stress $(8,37)$. Furthermore, a previous study demonstrated that free TIMP1 and magnetic nanoparticle-bound TIMP1 significantly reduced ROS production in HIV-infected neuroblastoma cells (38). In the present study, Ad-hTK1/hTIMP1 significantly reduced oxidative stress levels in myocardial I/R model rats and H/R-treated CMVECs. $\gamma$-H2A.X is H2A.X (a specialized histone H2A variant) phosphorylated at Ser 139 via ATM serine/threonine kinase and ATR serine/threonine kinase (39). $\gamma$-H2A.X is widely considered as a marker of DNA damage $(40,41)$. The results of the present study demonstrated that Ad-hTK1/hTIMP1 significantly decreased $\gamma$-H2A.X expression in $\mathrm{H} / \mathrm{R}$-treated CMVECs, indicating reduced DNA damage in H/R-treated CMVECs following transfection with Ad-hTK1/hTIMP1, which may be attributed to decreased oxidative stress. The aforementioned results demonstrated that co-expression of hTK1 and hTIMP1 genes may ameliorate myocardial $\mathrm{I} / \mathrm{R}$ injury via suppressing oxidative stress.

The present study demonstrated the protective effects of co-expression of hTK1 and hTIMP1 genes against myocardial I/R injury. However, a limitation of the present study was that only the preliminary protective effects of Ad-hTK1/hTIMP1, including promoting angiogenesis and suppressing oxidative stress, were investigated; therefore, the in-depth mechanisms underlying hTK1 and hTIMP1 expression in myocardial I/R injury require further investigation.

In conclusion, the present study demonstrated that co-expression of hTK1 and hTIMP1 genes displayed significant protective effects in myocardial I/R injury via promoting angiogenesis and suppressing oxidative stress. Therefore, co-expression of hTK1 and hTIMP1 may serve as a potential therapeutic strategy for myocardial I/R injury. 


\section{Acknowledgements}

Not applicable.

\section{Funding}

The present study was supported by the Sailing Fund of Fujian Medical University (grant no. 2018QH1136).

\section{Availability of data and materials}

The datasets used and/or analysed during the current study are available from the corresponding author on reasonable request.

\section{Authors' contributions}

$\mathrm{SH}$ performed the in vivo experiments and drafted the manuscript. MC performed the in vitro experiments. HY and KL analysed the data. YG and PZ designed the study and revised the manuscript. All authors read and approved the final manuscript.

\section{Ethics approval and consent to participate}

All animal experiments were conducted in accordance with the National Institutes of Health Guide for the Care and Use of Laboratory Animals (15), and approved by the Institutional Ethics Committee for Laboratory Animal Care of Fujian Provincial Hospital (approval no. K2019-01-038).

\section{Patient consent for publication}

Not applicable.

\section{Competing interests}

The authors declare that they have no competing interests.

\section{References}

1. Boateng $\mathrm{S}$ and Sanborn T: Acute myocardial infarction. Dis Mon 59: 83-96, 2013.

2. Benjamin EJ, Muntner P, Alonso A, Bittencourt MS, Callaway CW, Carson AP, Chamberlain AM, Chang AR, Cheng S, Das SR, et al: Heart disease and stroke statistics-2019 update: A report from the american heart association. Circulation 139: e56-e528, 2019.

3. Mehta LS, Beckie TM, DeVon HA, Grines CL, Krumholz HM, Johnson MN, Lindley KJ, Vaccarino V, Wang TY, Watson KE, et al: Acute myocardial infarction in women: A scientific statement from the American heart association. Circulation 133: 916-947, 2016.

4. Ni XQ and Hu ZY: Remifentanil improves myocardial ischemia-reperfusion injury in rats through inhibiting IL-18 signaling pathway. Eur Rev Med Pharmacol Sci 24: 3915-3922, 2020.

5. Chen Q, Zhou Y, Richards AM and Wang P: Up-regulation of miRNA-221 inhibits hypoxia/reoxygenation-induced autophagy through the DDIT4/mTORC1 and Tp53inp1/p62 pathways. Biochem Biophys Res Commun 474: 168-174, 2016.

6. Hausenloy DJ and Yellon DM: Myocardial ischemia-reperfusion injury: A neglected therapeutic target. J Clin Invest 123: 92-100, 2013.

7. Luan Y, Ruan Y, Wang T, Zhuan L, Wen Z, Chen R, Zhang Y, Cui $\mathrm{K}$, Yang J, Wang S, et al: Preserved erectile function in the aged transgenic rat harboring human tissue kallikrein 1. J Sex Med 13: 1311-1322, 2016.
8. Luan Y, Cui K, Tang Z, Ruan Y, Liu K, Wang T, Chen Z, Wang S and Liu J: Human tissue kallikrein 1 improves erectile dysfunction of streptozotocin-induced diabetic rats by inhibition of excessive oxidative stress and activation of the PI3K/AKT/eNOS pathway. Oxid Med Cell Longev 2020: 6834236, 2020.

9. Gao L, Bledsoe G, Yin H, Shen B, Chao L and Chao J: Tissue kallikrein-modified mesenchymal stem cells provide enhanced protection against ischemic cardiac injury after myocardial infarction. Circ J 77: 2134-2144, 2013.

10. Lorente L, Martín MM, Ramos L, Argueso M, Cáceres JJ, Solé-Violán J, Jiménez A, Borreguero-León JM, González-Rivero AF, Orbe J, et al: Persistently high circulating tissue inhibitor of matrix metalloproteinase-1 levels in non-survivor brain trauma injury patients. J Crit Care 51: 117-121, 2019.

11. Takawale A, Fan D, Basu R, Shen M, Parajuli N, Wang W, Wang X, Oudit GY and Kassiri Z: Myocardial recovery from ischemia-reperfusion is compromised in the absence of tissue inhibitor of metalloproteinase 4. Circ Heart Fail 7: 652-662, 2014.

12. Su YY, Li HM, Yan ZX, Li MC, Wei JP, Zheng WX, Liu SQ, Deng YT, Xie HF and Li CG: Renin-angiotensin system activation and imbalance of matrix metalloproteinase-9/tissue inhibitor of matrix metalloproteinase-1 in cold-induced stroke. Life Sci 231: 116563, 2019.

13. Kelly D, Squire IB, Khan SQ, Dhillon O, Narayan H, Ng KH, Quinn P, Davies JE and Ng LL: Usefulness of plasma tissue inhibitors of metalloproteinases as markers of prognosis after acute myocardial infarction. Am J Cardiol 106: 477-482, 2010.

14. Zhu P, Yu H, Huang S, Xiang H, Li F and Zheng W: Synergistic effect of a tissue kallikrein 1 and tissue inhibitor of matrix metalloproteinase 1 co-expression vector on the proliferation of rat vascular smooth muscle cells. Mol Med Rep 12: 5671-5678, 2015.

15. National Research Council (US) Committee for the Update of the Guide for the Care and Use of Laboratory Animals: Guide for the Care and Use of Laboratory Animals. 8th edition. National Academies Press, Washington, DC, 2011.

16. Yu HZ, Xie LD, Zhu PL, Xu CS and Wang HJ: Human tissue kallikrein 1 gene delivery inhibits PDGF-BB-induced vascular smooth muscle cells proliferation and upregulates the expressions of p27Kip1 and p2lCip1. Mol Cell Biochem 360: 363-371, 2012.

17. Zhu L, Xu C, Huo X, Hao H, Wan Q, Chen H, Zhang X, Breyer RM, Huang Y, Cao X, et al: The cyclooxygenase-1/mPGES-1/endothelial prostaglandin EP4 receptor pathway constrains myocardial ischemia-reperfusion injury. Nat Commun 10: 1888, 2019.

18. Hu S, Cao S, Tong Z and Liu J: FGF21 protects myocardial ischemia-reperfusion injury through reduction of miR-145-mediated autophagy. Am J Transl Res 10: 3677-3688, 2018.

19. Li W, Feng G, Gauthier JM, Lokshina I, Higashikubo R, Evans S, Liu X, Hassan A, Tanaka S, Cicka M, et al: Ferroptotic cell death and TLR4/Trif signaling initiate neutrophil recruitment after heart transplantation. J Clin Invest 129: 2293-2304, 2019.

20. Livak KJ and Schmittgen TD: Analysis of relative gene expression data using real-time quantitative PCR and the 2(-Delta Delta C(T)) method. Methods 25: 402-408, 2001.

21. Zheng QN, Wei XH, Pan CS, Li Q, Liu YY, Fan JY and Han JY: QiShenYiQi Pills ${ }^{\circledR}$ ameliorates ischemia/reperfusion-induced myocardial fibrosis involving RP S19-mediated TGF $\beta 1 /$ Smads signaling pathway. Pharmacol Res 146: 104272, 2019.

22. Lan TH, Huang XQ and Tan HM: Vascular fibrosis in atherosclerosis. Cardiovasc Pathol 22: 401-407, 2013.

23. Podesser BK, Kreibich M, Dzilic E, Santer D, Förster L, Trojanek S, Abraham D, Krššák M, Klein KU, Tretter EV, et al: Tenascin-C promotes chronic pressure overload-induced cardiac dysfunction, hypertrophy and myocardial fibrosis. J Hypertens 36: 847-856, 2018.

24. Scofield SLC, Dalal S, Lim KA, Thrasher PR, Daniels CR, Peterson JM, Singh M and Singh K: Exogenous ubiquitin reduces inflammatory response and preserves myocardial function 3 days post-ischemia-reperfusion injury. Am J Physiol Heart Circ Physiol 316: H617-H628, 2019.

25. Yin H, Chao L and Chao J: Nitric oxide mediates cardiac protection of tissue kallikrein by reducing inflammation and ventricular remodeling after myocardial ischemia/reperfusion. Life Sci 82: 156-165, 2008.

26. Yang Q, He GW, Underwood MJ and Yu CM: Cellular and molecular mechanisms of endothelial ischemia/reperfusion injury: Perspectives and implications for postischemic myocardial protection. Am J Transl Res 8: 765-777, 2016. 
27. Galaup A, Gomez E, Souktani R, Durand M, Cazes A, Monnot C, Teillon J, Le Jan S, Bouleti C, Briois G, et al: Protection against myocardial infarction and no-reflow through preservation of vascular integrity by angiopoietin-like 4. Circulation 125 : 140-149, 2012.

28. Yao YY, Yin H, Shen B, Chao L and Chao J: Tissue kallikrein and kinin infusion rescues failing myocardium after myocardial infarction. J Card Fail 13: 588-596, 2007.

29. Ling L, Hou Q, Xing S, Yu J, Pei Z and Zeng J: Exogenous kallikrein enhances neurogenesis and angiogenesis in the subventricular zone and the peri-infarction region and improves neurological function after focal cortical infarction in hypertensive rats. Brain Res 1206: 89-97, 2008.

30. Liu H, Chen B and Lilly B: Fibroblasts potentiate blood vessel formation partially through secreted factor TIMP-1. Angiogenesis 11: 223-234, 2008.

31. Lin KC, Yip HK, Shao PL, Wu SC, Chen KH, Chen YT, Yang CC Sun CK, Kao GS, Chen SY, et al: Combination of adipose-derived mesenchymal stem cells (ADMSC) and ADMSC-derived exosomes for protecting kidney from acute ischemia-reperfusion injury. Int J Cardiol 216: 173-185, 2016.

32. Mokhtari-Zaer A, Marefati N, Atkin SL, Butler AE and Sahebkar A: The protective role of curcumin in myocardial ischemia-reperfusion injury. J Cell Physiol 234: 214-222, 2018.

33. Li P, Stetler RA, Leak RK, Shi Y, Li Y, Yu W, Bennett MVL and Chen J: Oxidative stress and DNA damage after cerebral ischemia: Potential therapeutic targets to repair the genome and improve stroke recovery. Neuropharmacology 134: 208-217, 2018.

34. Rocca C, Boukhzar L, Granieri MC, Alsharif I, Mazza R, Lefranc B, Tota B, Leprince J, Cerra MC, Anouar Y and Angelone T: A selenoprotein T-derived peptide protects the heart against ischaemia/reperfusion injury through inhibition of apoptosis and oxidative stress. Acta Physiol (Oxf) 223: e13067, 2018.
35. Jiang M, Ni J, Cao Y, Xing X, Wu Q and Fan G: Astragaloside IV attenuates myocardial ischemia-reperfusion injury from oxidative stress by regulating succinate, lysophospholipid metabolism, and ROS scavenging system. Oxid Med Cell Longev 2019: 9137654, 2019.

36. Yao BJ, He XQ, Lin YH and Dai WJ: Cardioprotective effects of anisodamine against myocardial ischemia/reperfusion injury through the inhibition of oxidative stress, inflammation and apoptosis. Mol Med Rep 17: 1253-1260, 2018.

37. Zhang JJ, Bledsoe G, Kato K, Chao L and Chao J: Tissue kallikrein attenuates salt-induced renal fibrosis by inhibition of oxidative stress. Kidney Int 66: 722-732, 2004.

38. Atluri VS, Jayant RD, Pilakka-Kanthikeel S, Garcia G, Samikkannu T, Yndart A, Kaushik A and Nair M: Development of TIMP1 magnetic nanoformulation for regulation of synaptic plasticity in HIV-1 infection. Int J Nanomedicine 11: 4287-4298, 2016.

39. XiaoA,LiH,ShechterD,AhnSH,FabrizioLA,Erdjument-BromageH, Ishibe-Murakami S, Wang B, Tempst P, Hofmann K, et al: WSTF regulates the H2A.X DNA damage response via a novel tyrosine kinase activity. Nature 457: 57-62, 2009.

40. Zhang X, Mei Y, Wang T, Liu F, Jiang N, Zhou W and Zhang Y: Early oxidative stress, DNA damage and inflammation resulting from subcutaneous injection of sulfur mustard into mice. Environ Toxicol Pharmacol 55: 68-73, 2017.

41. Liu Y, Long YH, Wang SQ, Li YF and Zhang JH: Phosphorylation of H2A.XT ${ }^{\mathrm{y} 39}$ positively regulates DNA damage response and is linked to cancer progression. FEBS J 283: 4462-4473, 2016. International (CC BY-NC-ND 4.0) License. 